\title{
EN TORNO A LA CLASIFICACION DIALECTAL DEL PANFILIO
}

\begin{abstract}
In this paper the authors try to prove that the Greek dialect of Pamphylia is not a mixed dialect formed by an Achaean base and a Doric adstratum. The Doric features of the Pamphylian are archaisms, its innovations show that this dialect belongs to Eastern Greek (together with the Mycenaean, Epic Achaean, Protolesbian, Protoionic and Protoarcado-Cyprian) being the most conservative of this group. For this reason the Pamphylian is to be held as an intermediate dialect between Eastern Greek and Western Greek.
\end{abstract}

Las noticias que sobre Panfilia y el dialecto allí hablado proporcionan los antiguos son muy escasas y de pobre valor. Es de imaginar que el panfilio debió de ser un dialecto irreconocible como griego para los mismos griegos y Panfilia, sin duda, una zona olvidada; piénsese, si no, en la famosa cita de Isócrates, el pasaje del Panegírico en que el orador, al hablar de los griegos que habitan la costa asiática, menciona que se encuentran repartidos entre Sínope y Cnido ${ }^{1}$. Es ya en época muy reciente cuando Estrabón nos transmite que, a pesar de que en el pasado mítico de Panfilia figuran héroes fundadores como Mopso y Calcante, Selge fue fundada por segunda vez por los laconios, y Aspendo, por los argivos. He aquí, en traducción, los textos aludidos:

Dice Heródoto que los panfilios descienden de las mesnadas que seguían a Anfíloco y Calcante, gentes varias en mescolanza, que desde Troya les acompañaron; y que la mayor parte de ellos se quedó aquí (sc. Panfilia), mientras que unos cuantos se desperdigaron por muchos lugares de la tierra. Y Calino dice que Calcante acabó sus días en Claro, y que de las mesnadas que iban con Mopso, trasponiendo el Tauro, unos se quedaron en Panfilia y otros se repartieron por Cilicia, Siria e, incluso, Fenicia 2.

1 Isoc. IV 162.

2 Str. 668 (XIV 4, 3). 
Efectivamente, así lo dejó dicho de un modo explícito Heródoto en un texto utilizado por Estrabón y que dice así:

Lus panfilios ésos descienden de los que se desperdigaron, al regresar de Troya, juntamente con Anfíloco y Calcante 3.

Existian, pues, leyendas recogidas por Heródoto y Calino, según las cuales los asentamientos griegos en Panfilia tuvieron lugar en un período de tiempo posterior a la guerra de Troya, es decir, a finales del segundo milenio antes de Cristo. Las mencionadas leyendas, en efecto, se referían a la dispersión del contingente de tropas que, una vez tomada la ciudad de Príamo, fue en pos de Anfíloco, Mopso y Calcante.

Pero Estrabón en otros pasajes, que seguidamente presentamos, alude a una segunda fase de colonización griega de Panfilia, esta vez ya no a cargo de aqueos sino de dorios, y en el caso de Side, de eolios. Dice así, a propósito de Selge, primero, y de Aspendo, después:

Selge también, en principio, fue fundada por lacedemonios y aun anteriormente lo fue por Calcante 4 .

Respecto de la fundación de Aspendo, se expresa de este modo:

...Luego, un lago de grandes dimensiones, el Capria; y después, el río Eurimedonte; y si se remonta su curso en un trayecto de sesenta estadios, la ciudad de Aspendo, bastante poblada, fundación de argivos 5 .

De Side dice que era colonia fundada por gentes naturales de Cime ${ }^{6}$, o sea, colonia eolia.

Pues bien: los tratadistas modernos de los dialectos griegos tienen, por lo general, muy en cuenta estos datos que nos proporciona Estrabón. Así, casi todos incluyen al panfilio dentro del grupo del famoso "aqueo del sur", formando trío con el arcadio y con el chipriota y algunos reconocen que tiene puntos de contacto, más o menos acentuados según los autores, con dialectos dorios y eolios?

Meillet ${ }^{8}$ resta importancia a las concordancias del panfilio con el dorio e insiste en las isoglosas que lo vinculan al arcadio-chipriota. En

3 Hdt. VII 91.

4 Str. 570 (XII 7, 3).

5 Str. 667 (XIV 4, 2).

6 Str. 667; cf. Arr. An. I 26, 4; Scyl. GGM, I, p. 75, 101.

De los autores antiguos, Estrabón y Eustacio (GGM, II, p. 366, 852) son los únicos que hacen referencia a la presencia de dorios en Panfilia.

8 A. Meillet, "La place du pamphylien parmi les dialectes grecs", REG 21, 1908, p. 413 ss. 
cambio, Bechtel ${ }^{9}$ procede de manera totalmente contraria: estudia el dialecto panfilio dentro del segundo tomo de su magna obra, al lado de los dialectos dorios, y no junto al arcadio y el chipriota, y cree detectar en el panfilio una serie de rasgos que abogan por una estrecha relación de este dialecto con el cretense ${ }^{10}$.

Siete años más tarde, Ronconi " niega la existencia de dorismos en el panfilio. Kretschmer 12 acepta por lo general el planteamiento de Ronconi; disiente, empero, en la interpretación de hok $\alpha$, forma que considera un dorismo y se explicaría, al igual que otros dorismos, por la llegada de dorios a Panfilia con posterioridad a los aqueos ${ }^{13}$.

Metri trata de conciliar posturas: el núcleo del dialecto panfilio es para él el eolio en sentido amplio (es decir, eolio del norte y del sur, como él los llama, o "aqueo del norte» y "aqueo del sur", denominaciones más generalizadas), un eolio dentro del que caben el lesbio, tesalio, beocio, arcadio y chipriota; y sobre este núcleo se superponen elementos dóricos, algunos de los cuales son debidos a contactos recientes de Panfilia con Creta ${ }^{14}$.

Por último, en la más reciente publicación sobre el tema, la excelente tesis doctoral de Claude Brixhe ${ }^{15}$, utilísimo trabajo tanto por lo que se refiere a la edición de inscripciones como a la descripción de la lengua, nos topamos con una exposición sumamente cautelosa y tradicional de la relación del panfilio con los demás dialectos griegos. En efecto, el autor enumera las isoglosas que unen al pantilio con los dialectos aqueos, eolios y dorios y concluye que tales isoglosas son realmente reflejo de una historia muy compleja: colonización aquea hacia el 1200 a. J. C. y colonización doria en el periodo de la gran colonización (siglos vill y vil a. J. C.) ${ }^{16}$.

9 F. Bechtel, Die griechischen Dialekte, II, Berlín 1923, p. 796.

10 Cf. F. Bechtel, II, p. 797: "Eine kurze Analyse des pamphylischen Dialekts hat Meillet (Rev. des études gr. 21, 413 ff.) mitgeteilt. Meine eigene wird die Recht. fertigung dafür bringen, dass ich das Pamphylische an clas Kretische anschliesse.

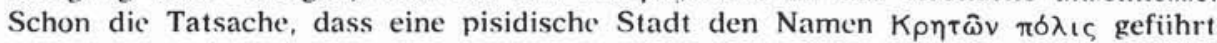
hat (Pol. V 72, 5) macht es offenbar, dass Kreter in diesen Teil Kleinasiens als Colonisten gelangt sind".

11 A. Ronconi, "Il dialetto della Panfilia", SIFG 8, 1930, p. 25 ss.

12 P. Kretschmer, "Zum pamphylischen Dialekt", KZ 33, 1895, p. 258 ss.

13 P. Kretschmer, "Literaturbericht für das Jahr 1930, Griechisch", Gl 21, 1933, p). 161-162. Cf. p. 162: "Wir dürfen in Kolonien gar keinen reinen Dialekt erwarten: hier fanden sich meist Auswanderer verschiedener Herkunft zusammen und legten sich im Laufe der Zeit mehrere Schichten von Kolonisten aufeinander».

14 P. Metri, "Il dialetto panfilio", RIL 87, 1954, pp. 79-117; cf. p. 112.

15 C. Brixhe, Le dialecte grec de Pamphylie, Documents et grammaire, París 1976.

$16 \mathrm{Cr}$. C. Brixhe, o. c., pp. 147-148: "La colonisation éolienne, qui vers la même énoque toucha Sidé, ne paraît pas avoir influencé le grec de Pamphylie». 
Nosotros vamos a revisar ahora esas isoglosas y a tratar de dilucidar si están o no bien trazadas.

Con los dialectos dóricos se señalan las siguientes isoglosas comu-

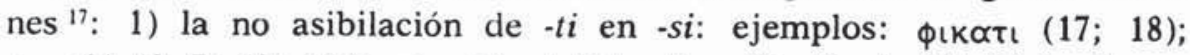
$\left.\pi \varepsilon \rho \tau(\iota)(3,7 ; 17 ; 18) ; \varepsilon \xi \alpha \gamma \bar{\delta} \delta \iota<{ }^{*} \varepsilon \xi \alpha \gamma \bar{\nu} v \tau \iota(3,16 ; 3,20) .2\right)$ La forma hok $\alpha(3,14)$, conjunción temporal, o, si se admite otra lectura, el uso

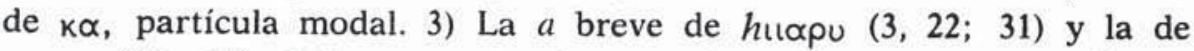

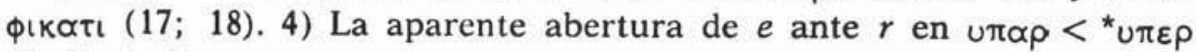
$(3,2) .5)$ La desinencia de tercera persona de plural de imperativo en

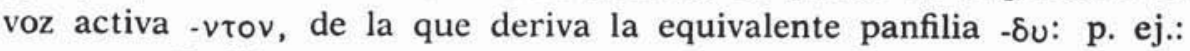
$\varepsilon \phi \iota \varepsilon \lambda \circ \delta \cup(3,11)$, que sería común al panfilio y al rodio. 6) Las frecuentes metátesis de líquidas, sobre todo de $/ r /$, p. ej.: $\pi \varepsilon \rho \tau(\iota)(3,7)$ en vez de $\pi \rho \varepsilon \tau(\mathrm{l})$, rasgo en el que coincidiría con el cretense, dialecto que, a título de ejemplo, ofrece la forma Schw. $179 \mathrm{~V} 44 \pi \circ \rho \tau$ en vez de * $\pi$ potl.

Pues bien, en cuanto a la primera (1), casi no es menester decir que se trata de un arcaísmo y que, por tanto, la coincidencia en la conservación de este rasgo y otros del mismo género por parte del panfilio y de los dialectos dóricos (además de otros dialectos griegos) no prueba sino que todos esos dialectos que lo conservan proceden del protogriego, lo cual no es decir nada nuevo. De ningún modo la conservación de un arcaísmo puede probar comunidad específica entre dialectos ${ }^{18}$.

Respecto de la segunda (2), en primer término habría que fijar definitivamente la lectura. Pero, en cualquier caso, si se admite $\delta \kappa \alpha$, la partícula modal $\kappa \alpha$ tiene todas las trazas de ser una de las formas de que disponían los dialectos para la expresión de la modalidad. Así se explica que aparezca también en beocio y no sólo en los dialectos dóri$\cos , \mathrm{y}$ que en arcadio junto a \&́v existan huellas de $\kappa \varepsilon^{19}$. En efecto, en un inteligente artículo, F. Bader ${ }^{20}$ pone en conexión las partículas $\kappa \varepsilon$,

17 La numeración que acompaña a ejemplos del panfilio corresponde a la mencionada edición de textos de Claude Brixhe. Formas de otros dialectos son citadas en referencia a otros repertorios bien conocidos, como IG, TAM (Tituli Asiae Minoris, Viena 1901 ss.), Schw. (E. Schwyzer, Dialectorum Graecarum exempla epigraphica potiora, Leipzig 1923), Masson o ICS (O. Masson, Les inscriptions chypriotes syllabiques, París 1961), Suppl. Epigr. (Supplementum Epigraphicum Graecum, Leyden 1923 ss.), etc.

18 F. R. Adrados, La dialectología griega como fuente para el estudio de las migraciones indoeuropeas en Grecia, Salamanca 1952.

19 Cf. E. Schwyzer, Griechische Grammatik, I, Munich 1938, p. 568. A. ThumbA. Scherer, Handbuch der griechischen Dialekte, II, Heidelberg $1959_{2}$, p. $140 \mathrm{~s}$.

20 F. Bader, "Lat. nempe, porceo et les fonctions des particules pronominales», $B S L 68,1973$, pp. 27-75. 
$\kappa \varepsilon \nu, \kappa \bar{\alpha}, \kappa \alpha \nu$ (de la que, según K. Forbes ${ }^{21}$, habría resultado \&̌́v por falso corte de $\varepsilon(\kappa \alpha \nu$, ouk $\alpha v)$ con el tema pronominal indoeuropeo *ke/o. Que aparezca, pues, $\kappa \propto$ en panfilio y en dialectos dorios sería simplemente coincidencia en una elección, lo que tampoco demuestra forzosamente que haya habido contacto estrecho - hasta el punto de ser interpretado como parentesco específico- entre los dialectos dorios y el panfilio. Simplemente coinciden en la elección de una de las varias posibilidades que la lengua madre ofrecía. Llegamos, así, a la conclusión de que el panfilio y los dialectos dóricos son dialectos griegos, lo que tampoco es mucho probar.

Y si, en vez de la lectura $8 \kappa^{22}$, entendemos que hay que leer 8 $\kappa \alpha$ podemos argumentar del mismo modo: cuando un mismo dialecto (el ático, por ejemplo) tiene formas temporales en $-\tau \varepsilon$ ( $\tau \delta ́ \tau \varepsilon, 8 \tau \varepsilon, \pi \delta \tau \varepsilon)$,

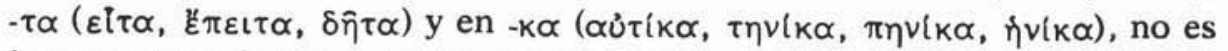
justo caracterizar $\delta_{k \alpha} \alpha$ como dorismo. Lo más razonable es, por el contrario, contar con las tres posibles terminaciones $(-\tau \varepsilon,-\tau \alpha, y-k \alpha)$ ya en griego común.

En efecto, para corroborar esta opinión, observemos el ejemplo siguiente: dado que - $\tau \alpha$ está, sobre todo, extendido en lesbio y $\varepsilon l$ es la forma de la conjunción condicional en jónico-ático, arcadio y micénico, ¿sería correcto interpretar $\varepsilon i \tau \alpha$ como un híbrido resultado de la mezcla de un elemento jónico con otro eólico? De ningún modo.

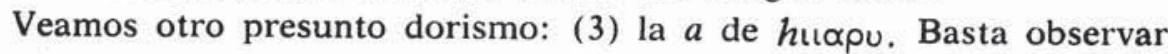

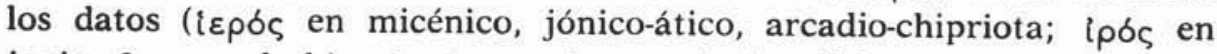

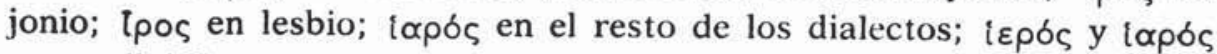
en tesalio) ${ }^{23}$ para concluir que de nuevo estamos ante un caso de elección y que hubo un estadio común en que coexistían las tres posibilidades. No se puede, pues, explicar la $a$ del panfilio hı๙apu a través de la del dorio lapós, sino que una y otra proceden de una forma * ${ }^{*} \rho \delta \varsigma$ que coexistió con *iepós - así ocurre en tesalio-. Por tanto, también por lo que a este rasgo se refiere alcanzamos la conclusión de que pan-

21 J. Forbes, "The Relations of the particle \& $v$ with $\kappa \varepsilon(v) \kappa \alpha \kappa \alpha v$ " Gl 37, 1958, pp. 179-182.

22 Sobre la dificultad de la lectura de la línea 14 de la gran inscripción de la acrópolis de Silion, cf. W. M. Ramsay, JHS 1, 1880, p. 255 ss.; H. Roel, Inscriptiones Graecae antiquissimae praeter Atticas in Attica repertas..., Berlín 1882; A. Bezzenberger, SGDI, I, Göttingen 1884, p. 365 ss.; R. Meister, Sächs. Ber. 56, 1904, p. 37; E. Hermann, Die Nebensätze in den griechischen Dialektinschriften, Leipzig 1912, p. 305; A. Scherer, Handbuch der griechischen Dialekte, II, Heidelberg 1959, p. 192. lisch».

Cf. A. Thumb-A. Scherer, o. c., II, p. 55: "Die Form $t \varepsilon \rho \delta \varsigma$ ist auch thessa- 
filio y dialectos dóricos proceden del protogriego, pero no podemos probar que haya existido comunidad específica entre ellos.

Lo mismo cabe decir de la $a$ de $ф ı к \propto \tau \iota$, resultado, como es sabido, del tratamiento de una sonante nasal $n$ : en micénico coexisten dos resultados distintos del tratamiento de $n: a$ y $o$, por ejemplo: pe-mo $\left(\sigma \pi \varepsilon \varepsilon_{\rho}\right)$ y pe-ma $(\sigma \pi \varepsilon \dot{\varepsilon} \rho \alpha)$. Pero, además, por doquier en los dialectos del primer milenio se encuentra uno con dobletes similares. Así: « $p \mu \alpha$,

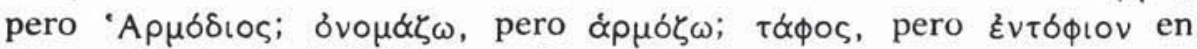
Cirene (Notiz. Arch. 4, 96); en ático, al lado de $\sigma \pi \varepsilon \rho \mu \alpha$ tenemos $\sigma \pi \varepsilon \rho$ $\mu \circ \lambda \delta$ бos, $\alpha i \mu \alpha$ - frente a $\alpha i \mu o-$ como primeros elementos de compuesto, etc. ${ }^{24}$ No vale, por tanto, la pena detenerse en este punto por más tiempo. Es claro que tampoco este rasgo sirve para demostrar parentesco específico (y no genérico, que de ése sí estamos convencidos) entre el panfilio y los dialectos dóricos.

Pasamos al punto siguiente (4); la abertura de $e$ en $a$ en $u \pi \propto \rho<$ * $\dot{i} \pi \hat{\varepsilon}$. La abertura de $e$ breve en $a$ en contacto con $r$ es un fenómeno de fonética general. En los dialectos griegos del primer milenio, se atestigua

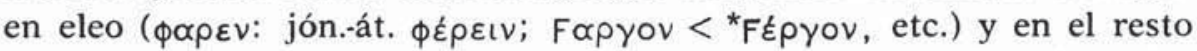
de los dialectos nordoccidentales, aunque siempre con inconsistencias

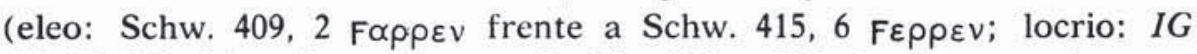

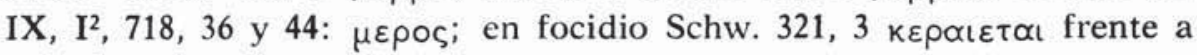
Schw. 321, $1 \phi \alpha \rho \varepsilon v$, etc.). Como en panfilio, aparentemente, también en Epidauro tropezamos con la forma u $\pi \alpha \rho$ (Schw. 109, 132) en vez de * $\dot{\pi} \varepsilon \rho, 0$, más exactamente, en vez de ü $\pi \varepsilon \rho$, pues en la inscripción

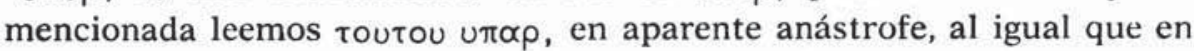
la inscripción panfilia, en la que la lectura del contexto de ur $\propto \rho$ es:

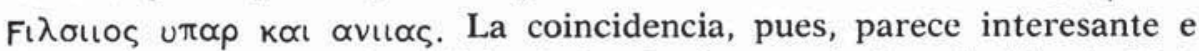
ilustradora, ya que el análisis de los hechos en la inscripción de Epidauro revela que se trataría, en todo caso, de un fenómeno esporádico, pues í $\pi \dot{\varepsilon} \rho$ se lee en ella dos veces $(1,82)$; lo mismo podemos deducir de la situación de la secuencia er en panfilio: aislada y esporádicamente aparece como $\alpha \rho$ (u $\pi \alpha \rho$ ), pero, por lo demás, se mantiene perfectamente: así $\pi \varepsilon \rho \tau(3,7)$; $\gamma \varepsilon \rho \alpha \varsigma(3,22)$, etc. Ahora bien, basta leer con detención el mencionado pasaje de la inscripción de Epidauro para cercio-

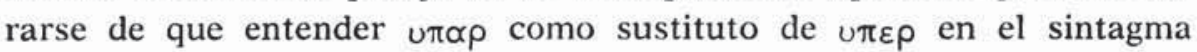

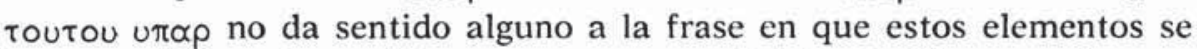
integran. Más vale considerar que u $\pi \propto \rho$ es el indeclinable $̋ \pi \alpha \rho$ famoso que se opone a $\delta_{v} \alpha \rho$. Luego huelga hablar de $u \pi \alpha \rho$ como rasgo común

24 Véase el espléndido artículo de F. Bader, "De mycénien matoropuro, arepozoo

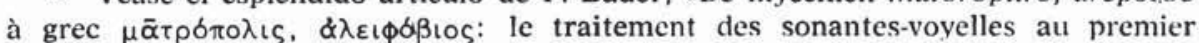
millénaire», Minos 10, 1970, pp. 7-63. 
del dorio y del panfilio. Tampoco, por consiguiente, creemos nosotros que u $\pi \alpha \rho$ sea evolución de $u \pi \varepsilon \rho$, sino, tal vez, forma colateral de húper con grado cero: hupr (cf. mic. upar-/upor-).

Vayamos ahora al punto (5), el de la desinencia de tercera persona de plural del imperativo en voz activa $-v \tau o v$. Digamos, no obstante, previamente que, para plantear como es debido esta isoglosa, habría que decir: isoglosa que une al panfilio con una inscripción rodia ${ }^{25}$ en la que aparece la forma $\pi \propto \rho \propto \kappa \alpha \lambda \varepsilon \cup \nu \tau o v$, tercera persona de plural del imperativo activo provista, como es notorio, del morfema $-v \tau o v$, el mismo del que deriva el morfema equivalente panfilio - $\delta$. Podemos añadir que en Fasélide, colonia fundada por Rodas hacia el 700 a. J. C. en la costa oriental de Licia, cerca de la actual Tekirova, o sea, localidad muy próxima a Panfilia, se lee en un tratado ${ }^{26}$ concertado entre

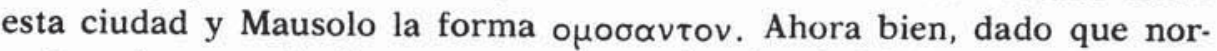
malmente en rodio la tercera persona de plural del imperativo activo

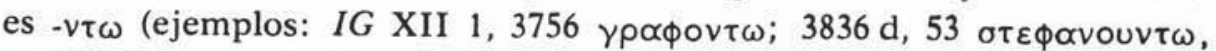
etcétera) y, en cambio, donde normalmente aparece - $v \tau o v$ es en lesbio

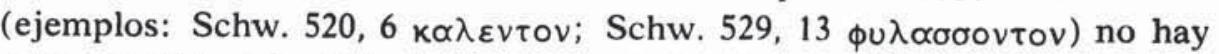
más remedio, a la vista de los hechos, que suponer bien que - $\nu$ tov proceda de $-v \tau \omega \nu$ por abreviamiento, bien que $-\nu \tau \omega \nu$ y $-\nu \tau o v$ hayan coexistido, habiéndose formado $-v \tau o v$ analógicamente a la oposición $\varepsilon \gamma v \omega /$ हैyvov. En cualquier caso, queda bien claro, en primer lugar, que no debemos conectar sin más al panfilio con los dialectos dorios basándonos en este rasgo de la desinencia de tercera persona de plural del imperativo en voz activa - $v \tau o v$ (panfilio: $-\delta u$ ). En segundo término, los datos nos inclinan a pensar que estamos ante un caso de elección o de desarrollos paralelos. Sólo así se entiende que en un mismo dialecto (el rodio) coexistan ambos morfemas, $-v \tau \omega$ y $-v \tau o \nu$.

Pasamos, pues, a tratar el último punto de contacto que se establece entre el panfilio y el grupo dialectal dórico, el número (6), el de las frecuentes metátesis de las líquidas, rasgo que, una vez más, no es común al panfilio y los dialectos dóricos en general, sino al panfilio y al cretense en particular.

Efectivamente, no se puede negar que tanto en panfilio como en cretense nos topamos a menudo con formas en que ha tenido lugar metátesis, como, por ejemplo, en panfilio $\pi \varepsilon \rho \tau$ - en vez de $\pi \rho \varepsilon \tau-(18 ; 19)$,

25 IG XII 1, 3751.

26 TAM II 3, 1183. Cf. P. Metri, o. c., p. 96: "Una spiegazione soddisfacente sull'origine di tali desinenze non è stata ancora trovata... Due esempi di - $v$ tov anche a Rodi... e Phaselis... dove possono essere eolismi». 
que recuerda la forma de la preposición cretense $\pi \circ \tau_{\iota}$ (Schw. $179 \mathrm{~V}$ $44 \pi \circ \rho \tau \iota \propto \mu \rho \lambda\llcorner\circ \mu \varepsilon v \alpha)$ frente a la forma esperada $\pi \rho \circ \tau l$.

Pero la metátesis en sí es un hecho de fonética general que se registra en todo el ámbito del griego y, tratándose de $r$, el proceso es aún más frecuente y diluido. En efecto, ejemplos de metátesis de $r$ se encuentran en micénico, arcadio, argivo, ático, etc. La $r$ es extraordinariamente móvil y en un mismo estado de lengua y dentro de un mismo

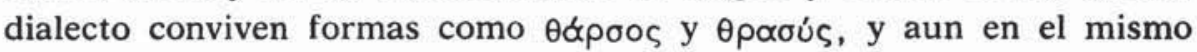

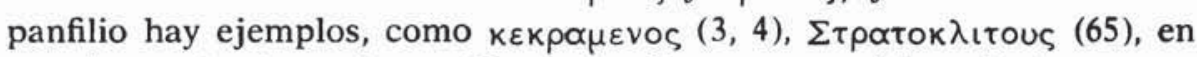
que la metátesis no ha tenido lugar. $\mathrm{Y}$ aunque es bien cierto que en

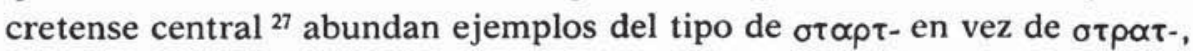

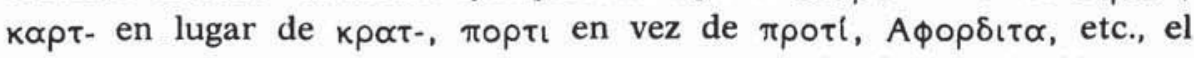
hecho de que en la misma Creta haya constancia de la solución contraria y aun zonas en que una y otra coexisten nos obliga a ser cautos; tanto más cuanto que en micénico conviven $\theta \rho \delta$ vo- (to-ro-no-) y $\theta \delta \rho v o s$

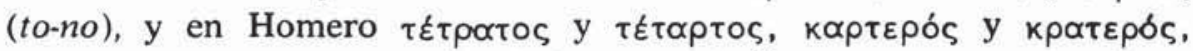

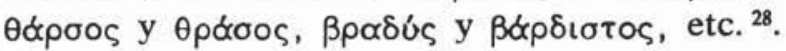

Resumiendo, pues, lo que hasta ahora hemos venido exponiendo, podemos decir que no hay una sola innovación (el único rasgo que permite probar parentesco específico en dialectología) que sea común al panfilio y a la generalidad de los dialectos dóricos, y que, por consiguiente, no podemos caracterizar al panfilio como dialecto dórico. Es decir: mientras que nadie vacilaría en considerar al jonio minorasiático, al de las Cícladas, al de Eubea y al ático miembros de un mismo grupo dialectal, el jónico-ático, porque comparten una serie de innovaciones (paso de alfa larga a eta: $\bar{\alpha}>\eta$; formas especiales de los pronombres personales: $\eta \mu \varepsilon \hat{\imath}$, etc.), nada similar se deduce al comparar al panfilio con los dialectos dóricos, o grupo dialectal dórico, si se prefiere.

Y una vez hemos llegado a este punto, conviene insistir en un hecho que con harta frecuencia se olvida: el que el dialecto panfilio coincida con el grupo dialectal dórico en determinados rasgos no quiere decir

27 E. Kieckers, Die lokalen Verschiedenheiten im Dialekte Kretas, Marburgo 1908 , p. 52 ss. Para Bechtel, el hecho de que coincidan cretense y panfilio en la metátesis de $r$ es una prueba del carácter dorio del panfilio. Luria, en cambio, interpreta que la metátesis de $r$ en cretense es una huella del sustrato aqueo. Cf. F. Bechtel, o. c., II, p. 809: «Dies ist der zweite Zug durch den das Pamphylische in nähere Beziehung zu dem Kretischen gebracht wird». S. Luria, Klio 37, 1959, p. 7 ss.; cf. p. 14. Cl. Brixhe atribuye cautamente el fenómeno de la metátesis de $r$ al sustrato anatolio; cf. $\mathrm{Cl}$. Brixhe, o. c., p. 62: "...Si sur ce point le pamphylien doit quelque chose à un autre parler, c'est, nous semble-t-il, au substrat anatolien».

28 P. Chantraine, Grammaire homérique (Phonétique et Morphologie), París 1942, pp. 23-24. 
nada ni prueba, desde luego, comunidad específica entre estos dialectos. Para que ésta pueda ser demostrada hay que probar previamente que los rasgos en cuestión son innovaciones. De esta forma, cuando para caracterizar al dialecto panfilio se señala que con los dialectos dorios comparte la no asibilación de $/ t /$ ante $/ i$ / en final absoluto (ejemplos:

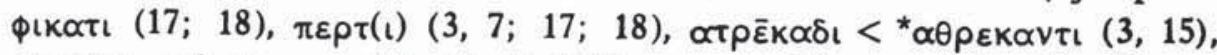
$\left.\varepsilon \xi \alpha \gamma \bar{o} \delta \iota<{ }^{*} \varepsilon \xi \alpha \gamma \omega \nu \tau \iota(3,16 ; 3,20)\right)$, no se está caracterizando al panfilio como dialecto afín a los dialectos dorios, sino afirmando sencilla y llanamente que tanto el panfilio como los dialectos dorios son dialectos griegos. Lo mismo estaríamos haciendo si afirmásemos que el panfilio tiene en común con todos los dialectos griegos (jónico-ático exclui-

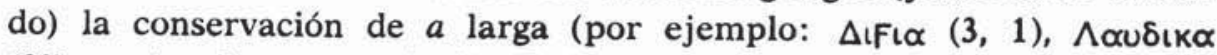
(91), etc.) y la pronunciación $[u]$ y no $[\ddot{i}]$ de $u$, tal como puede inferirse de algunas grafías: Eutuxus (82), Eutouxou (112), youv $\alpha$ (78; 151). Otros arcaísmos hay en panfilio que coinciden con mayor o menor número de dialectos griegos o incluso con ninguno de los que nos han transmitido las inscripciones del primer milenio a. J. C. Veamos algunos de ellos: la conservación de aspiración inicial: hıœpoı $(3,9), h \propto(3,15)$; la ausencia de $-v$ efelcística: $\pi \varepsilon \rho \tau \varepsilon \delta \omega \kappa \varepsilon$ เ५ (17); la falta de artículo: acerca del sintagma $\cup \beta O \lambda \bar{\varepsilon} \mu \varepsilon v \cup \varsigma(3,13)$ ha tratado últimamente con gran tino e inteligencia García Teijeiro, a cuyo trabajo sobre esta cuestión remitimos ${ }^{29}$; el empleo de $\varepsilon \xi$ ante vocal y ante consonante, como ocurre también en chipriota: $\varepsilon \xi \varepsilon \pi \iota \tau \bar{\varepsilon} \rho \iota \alpha(3,4), \varepsilon \xi$ $\delta \varepsilon \phi \cup \sigma \varepsilon \lambda \propto(3,19)$; el empleo del sufijo de agente - $\tau \varepsilon \rho-$ y no - $\tau \bar{\alpha}$ como en

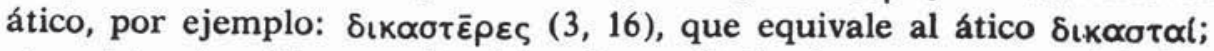
el aoristo atemático no sigmático $h_{\iota \kappa \propto \iota}(3,9)$; los compuestos del tipo de $A \theta \propto v \propto \delta \omega \rho \propto$ (47), $\Theta \alpha v \propto \delta \omega \rho \nu \varsigma$ (26), $\Theta \alpha v \propto \delta \omega \rho o u ~(49), ~ \Theta \alpha v \alpha \delta \omega \rho \nu$ (155), etcétera; formas léxicas, como $\Delta \mathrm{t} / \alpha(3,1)$ (que recuerda las micénicas

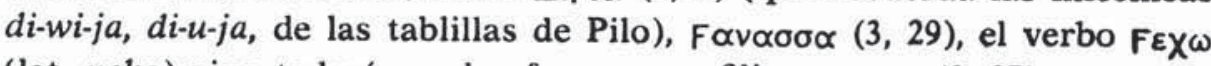
(lat. ueho) vivo todavía en las formas panfilias (54), que nos hacen pensar de inmediato en la forma del chipriota $\varepsilon F \varepsilon \xi \varepsilon$ (245 Masson) ${ }^{30}$, el sustantivo d́yós glosado por Hesiquio como equivalente de $\eta \gamma \varepsilon \mu \omega v$, tal vez el timbre radical $e$ del nombre de Apolo en panfilio: $A \pi \varepsilon \lambda \circ v \alpha(3,30), A \pi \varepsilon \lambda \alpha \delta \circ \rho \cup \varsigma$ (129), en que coincide este dialecto con el laconio, cretense, corintio, chipriota y otros.

Queda, pues, claro que de nada nos valen los arcaísmos del panfilio a la hora de buscarle el parentesco específico; estos arcaísmos aparecen también en muchos otros dialectos (y no sólo los dóricos) sin que por

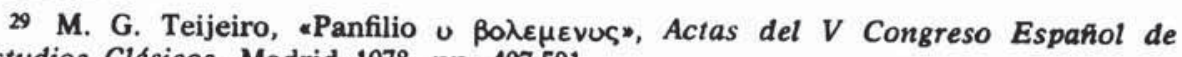
Estudios Clásicos, Madrid 1978, pp. 497-501.

30 O. Masson, Les inscriptions chypriotes syllabiques, París 1961.

LI, $10^{\circ}-2$ 
ello estemos autorizados a deducir de este hecho la pertenencia de esos dialectos y del panfilio al mismo grupo dialectal; pertenecen -eso síal bloque griego (parentesco genérico), son todos ellos dialectos griegos, pero nada más podemos inferir.

Eliminado, por consiguiente, el riesgo de establecer a la ligera un parentesco específico del panfilio con el grupo dialectal dórico, pasemos ahora a examinar los rasgos en que se ha querido fundar una vinculación genética estrecha entre el panfilio y el grupo eolio, o más concretamente, el lesbio. Son cuatro, a saber: el tratamiento por labial de una

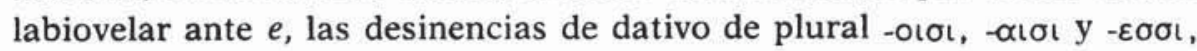
la flexión atemática de los verbos contractos y la tercera persona de plural del imperativo activo y medio-pasivo en - $v \tau o v$ y - $\sigma \theta 0 \nu$ (panfilio $-\delta \cup$ y - $\sigma \delta \cup$; lesbio - $v \tau o v$ y $-\sigma \theta \circ v$ ). Como de este último rasgo ya hemos tratado a propósito de la coincidencia del panfilio con el rodio ${ }^{31}$, abordaremos tan sólo los tres restantes.

Del presunto tratamiento por labial de una labiovelar ante $e$ en panfilio se aducen testimonios escasos y poco probatorios: nombres pro-

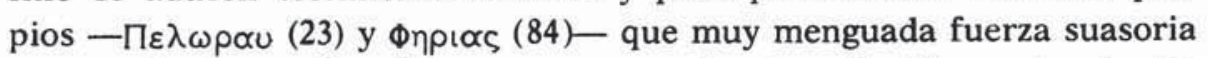
poseen frente a ejemplos que argumentan la solución contraria (es decir, el tratamiento por apical de una labiovelar ante $e$ ), como $\pi \varepsilon \delta \varepsilon<$ ${ }^{*} \pi \varepsilon \nu \tau \varepsilon(3,5), \phi \iota \lambda \alpha \delta \varepsilon \lambda \phi \phi \circ$ (146), $\phi ا \lambda \alpha \delta \varepsilon \lambda \lambda \phi \propto \iota$ (154) ${ }^{32}$.

En cuanto a las desinencias de dativo de plural, en panfilio, efecti-

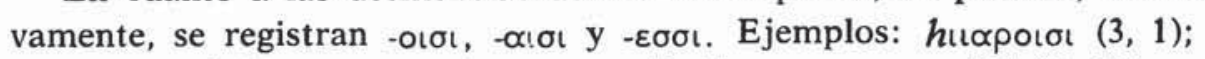

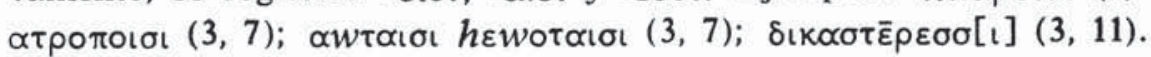

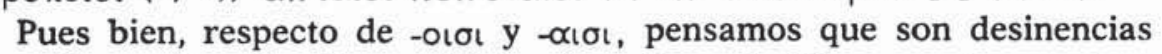

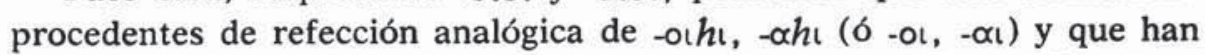
existido en todos los dialectos al lado de -oıs y - a s. En panfilio estaríamos, pues, ante un caso de elección. En cuanto a la extensión de $-\varepsilon \sigma \sigma l$, que, como es sabido, existía ya en protogriego en los temas en -s( $\gamma \varepsilon \dot{\varepsilon} \varepsilon \sigma \sigma \iota)$ y que suplanta a $-\sigma \iota$ en los temas en consonante no sólo en dialectos eolios, sino también en délfico, locrio, eleo, cireneo y dialectos de colonias de Corinto, parece lógico y prudente pensar que se trata de una innovación que brota independientemente en distintas áreas. Así lo cree también Claude Brixhe ${ }^{33}$.

31 A. Thumb lo consideraba eolismo del rodio; cf. A. Thumb, Gr. Dial, I, p. 191.

32 Cf. Cl. Brixhe, o. c., p. 92: «Il parait donc imprudent de rattacher les formes comportant un traitement labial à une prétendue strate éolienne du dialecte».

$33 \mathrm{Cl}$. Brixhe, o. c., p. 103: «on peut se demander si nous n'avons pas affaire à une innovation indépendante dans chaque domaine». Cf., sin embargo, P. Metri, o. c., pp. 94 y 109. 
Sobre la posible interpretación de $\beta \circ \lambda \bar{\varepsilon} \mu \varepsilon v \cup \varsigma(3,13)$ como participio de $\beta \circ \lambda \bar{\varepsilon} \mu \alpha \iota$, flexión atemática del $\beta \circ \lambda \varepsilon \mu \alpha_{\iota}$ esperado, que, a nuestro juicio, es distinto de $\varepsilon \beta 0 \lambda \alpha \sigma \varepsilon \tau u(3,8)$ en formación y significado, cabe puntualizar, en primer término, que rastros de flexión atemática se hallan en dialectos no eolios (eleo, argivo, dialecto de Cirene) mientras que, por otro lado, hay ejemplos de flexión temática de contractos en un dialecto eolio, el lesbio. Ello nos hace pensar que estamos ante un fenómeno de elección. En segundo lugar, si $\beta ం \lambda \bar{\varepsilon} \mu \varepsilon v \cup \varsigma$ no está formado, como pensamos, sobre $\beta \bar{\partial} \lambda \epsilon$, habrá que revisar esta cuestión de la flexión atemática de los contractos en panfilio.

Por consiguiente, nada de eolismos en panfilio. No nos sentimos obligados a postular parentesco específico de este dialecto con el grupo dialectal eolio, ni, en concreto, con el lesbio ${ }^{34}$.

Habrá, pues, que seguir indagando en los datos lingüísticos que ofrece el panfilio para poder llegar, de este modo, a averiguar con qué dialecto o dialectos está específicamente emparentado.

El panfilio, como todo dialecto o todo estado de lengua, contiene en sí arcaísmos (rasgos lingüísticos conservados), elecciones (rasgos elegidos o seleccionados de entre varios de la misma especie que poseía el estado de lengua anterior) e innovaciones (rasgos evolucionados, transformados respecto de los que fueron su origen en la lengua madre).

Pues bien, dado que ya hemos examinado los arcaísmos, veamos ahora algunas elecciones del panfilio que hasta este momento no hayamos considerado ( $y$ no olvidamos que la coincidencia en elecciones tampoco prueba parentesco específico entre los dialectos que las comparten): en el dialecto que nos ocupa, frente a la forma $\Delta, \delta \delta \omega p o s$ que esperaríamos, encontramos $\Delta \mathrm{L} F \mathrm{t} \delta \omega \rho \cup \varsigma$ (158), $\Delta \mathrm{L} F \mathrm{l} \delta \omega \rho \cup(129 ; 135 ; 162)$, $\Delta \mathrm{L} i \delta \omega$ pous (23), etc. Dado que en chipriota contamos con el mismo tipo de compuesto sólo que provisto en su primer elemento de un dativo en -ei (ICS 327, 8; 352, 1 y $3 \Delta$ เF y en jónico-ático coexisten $\Delta \iota \sigma \omega \tau \eta p ı \alpha^{35}$ (dativo en $-i$ en el primer elemento) y $\Delta \iota \varepsilon เ \tau \rho \varepsilon \phi \eta \varsigma^{36}$ (dativo en -ei), habrá que concluir que el dativo de los atemáticos en - $i$ es en panfilio, al igual que en otros dialectos, una elección; o, dicho de otro modo: el dativo ha extendido y especializado $-i$ como desinencia de dativo de singular de un atemático, aunque en un estado de lengua anterior coexistían las desinencias $-e i$ e $-i$ en la misma función casual.

34 Cf. Cl. Brixhe, o. c., p. 146: «Nous avons vu qu'aucun des ces traits ne reflétait nécessairement des rapports entre les deux domaines dialectaux».

35 IG 22 1008, 21.

36 SGDI 5400 b, 12 (Ceos, s. v a. J. C.). 
Veamos otros casos de elección: la forma del panfilio $\alpha v h \alpha \gamma \lambda \bar{\varepsilon} \sigma \theta \overline{0}$

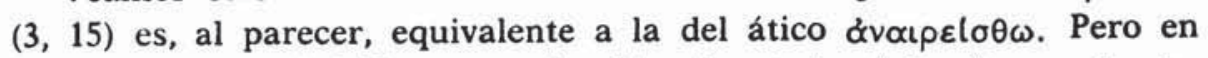

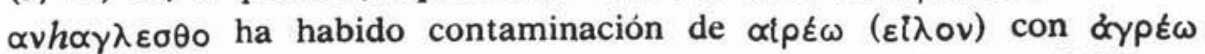
(verbo equivalente a $\alpha i \rho \xi \omega$, por su significado, en algunos dialectos, como el eleo, el lesbio $\mathrm{y}$, tal vez, el micénico). Como se da el caso de que en cretense central encontramos formas del tipo de $\alpha \iota \lambda \varepsilon \theta \varepsilon \iota^{37}$, $\alpha v \propto \alpha_{\imath} \lambda \varepsilon \theta \theta \alpha{ }^{38}$, que, evidentemente, han resultado del cruce de $\alpha i p \varepsilon \omega \mathrm{y}$ $\varepsilon\left(\lambda \circ \nu, y\right.$, por otro lado, la formación del tesalio $\varepsilon \phi \propto v \gamma \rho \varepsilon v \theta \varepsilon \iota{ }^{39}$ presupone cruce de $\alpha i \rho-(\alpha i \rho \varepsilon \omega,-\eta \mu \mathrm{t})$ y $\delta \gamma \rho-(\delta \gamma \rho \varepsilon \omega,-\eta \mu \mathrm{t})$, al igual que la

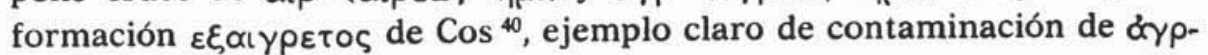

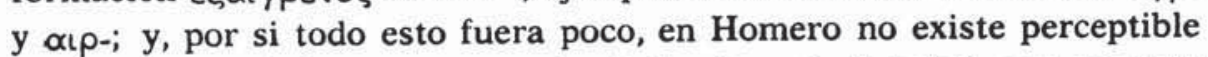

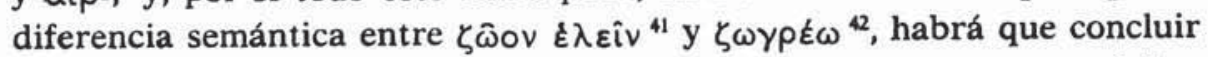
que la forma del panfilio es un caso de elección en que no se especializa una sola de entre varias formas sino una de compromiso entre ellas.

Veamos otro caso: encontramos en panfilio compuestos del tipo de A $\pi \varepsilon \lambda \alpha \delta \delta$ o $\cup \varsigma$ (129), П $\varepsilon \lambda \alpha \delta \omega \rho \cup \varsigma$ (117), cuyo primer elemento acaba no en $-o$ como cabría esperar, sino en $-\alpha^{43}$. Ello significa que el panfilio en este caso extiende la solución $-n>a$ después de una época anterior de vacilación $-0 /-a$, de la que quedan huellas en los dobletes $\alpha\{\mu o-/ \alpha \mid \mu \alpha-$,

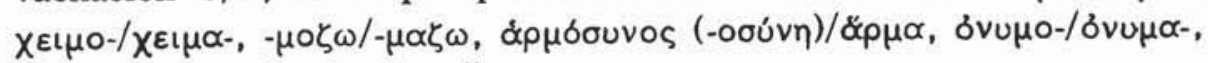
etcétera, en todo el griego ${ }^{44}$.

Otro caso de elección en panfilio es la partícula vı (por ejemplo,

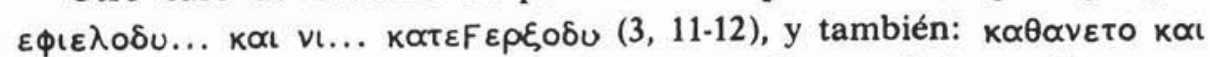
vi $(3,17))$, enclítica que se emplea para reforzar el imperativo y es similar en función a la partícula vu (vuv) que encontramos en chiprio$\mathrm{ta}^{45}$, beocio ${ }^{46}$ y Homero ${ }^{47}$. Son partículas intensivas que tienen correlato en varias lenguas indoeuropeas: $v \iota$ en frigio, $n u$ en hitita (donde sirve para iniciar una frase) ${ }^{48}, n u$ en sánscrito y avéstico, etc. La enclítica vi, dentro del mismo griego, aparece también en conglomerados pronomi-

37 Schw. 179 II 21.

38 Schw. 179 V 24.

39 Schw. 590, 41.

40 SGDI IV 3, p. 564.

41 Il. 21,$102 ; 6,38$.

42 Il. $6,46$.

43 Cl. Brixhe, o. c., pp. 135-136.

4 Cf. F. Bader, Minos 10, 1970, p. 7 ss.

45 ICS 217, 6; 16. Cf. F. Bechtel, o. c., II, p. 819. P. Metri, o. c., p. 97.

46 Schw. 523, 165. Cf. P. Metri, o. c., p. 97.

47 Cf. C. J. Ruijgh, L'élément achéen dans la langue épique, Assen 1957, p. 64: *En pamphylien, on trouve $k \alpha l v t$ introduisant impératif; $v \iota$ a donc la même valeur que vux.

48 E. Benveniste, Hittite et indo-européen, París 1962, p. 71. H. Kronasser, Vergleichende Laut- und Formenlehre des Hethitischen, Heidelberg 1956, p. 153. 
nales del tipo de beocio $\tau \alpha v$, arcadio ${ }^{50} \tau \omega v$ ı (cf. también arcadio $\tau \propto เ v v v<{ }^{*} \tau \propto \iota \sigma v{ }^{51}$ y chipriota $\left.{ }^{52} o-n u\right)$ y en adverbios de tiempo, como $\eta v(k \alpha, \tau \eta v(k \propto, \pi \eta v(k \alpha$. De todo lo dicho se infiere que $v \iota$ en panfilio es un caso clarísimo de elección.

Veamos otro: la preposición y preverbio $\pi \varepsilon \rho \tau$, que aparece en $\pi \varepsilon \rho \tau^{\prime} \iota \rho \varepsilon v \iota ~(3,7), \pi \varepsilon \rho \tau \varepsilon \delta \bar{o} \kappa \varepsilon$ (17), $\pi \varepsilon \rho \tau \varepsilon \delta \omega \kappa \varepsilon$ (18). Esta forma remonta a *$\pi \rho \varepsilon \tau \iota$, grado $e$ de $\pi \rho \circ \tau$. De * $\pi \rho \varepsilon \tau \iota$ derivan: por metátesis, $\pi \varepsilon \rho \tau \iota$ del panfilio; por asibilación de $-t i$ en -si y apócope de $-i$ en proclisis, la forma $\pi \rho \varepsilon ́ s$ que un gramático ascribe al lesbio ${ }^{53}$, y la primera parte

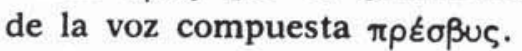

Podríamos seguir enumerando y comentando elecciones, pero para nuestro propósito las mencionadas son suficientes, pues es claro que lo que sobre todo nos interesa es establecer el capítulo de innovaciones y examinarlas una a una para poder hablar de parentesco específico entre el panfilio y aquel o aquellos dialectos griegos con los que las comparta.

Ahora bien, no olvidemos que para ese fin es menester fechar las innovaciones en cuestión. Y aquí, curiosamente, radica una de las grandes sorpresas que experimenta el dialectólogo en el momento de catalogar genéticamente al dialecto panfilio: la mayor parte de las innovaciones de este dialecto son compartidas por la koiné. Veamos algunas de las más significativas:

Es bien conocido el hecho de que en panfilio las desinencias -ios e -ion, independientemente de su origen (es decir: ya sean de nominativos o acusativos de sustantivos o adjetivos de la declinación temática, ya de genitivos de los temas en - $i$ o en -es) experimentan la evolución siguiente: -iiio- $>-i i u_{-}>-\overline{-}$ - (notado $\varepsilon t$ a partir del siglo III a. J. C.).

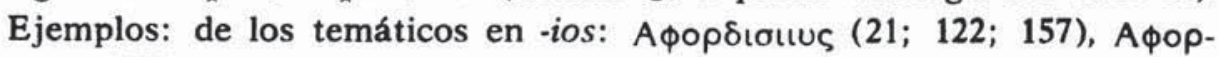

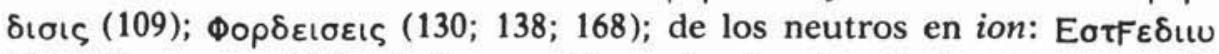
(8; 11); $\varepsilon \rho \varepsilon \mu \nu$ ( 17$)$; de los genitivos de singular de los temas en $-i$ :

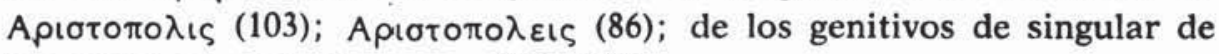

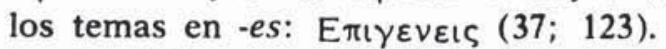

Pues bien, en griego helenístico esa evolución está atestiguada a partir del siglo III a. J. C. para los nominativos y acusativos acabados en

49 IG VII 1739, 5.

50 Schw. 656, 36; 657, 14.

51 Schw. 657, 30; cf. también, en arcadio, el adverbio $\tau \varepsilon ı \delta \varepsilon v \cup$ (Schw. 651).

52 ICS $215 \mathrm{~b}, 1 ; 216 \mathrm{~b}, 1$.

53 Cf. A. Thumb-A. Scherer, o. c., II, p. 108. R. Maister, Die griechischen Dialekte, I, Göttingen 1882, p. 44; Ch. Lambert, Etude sur le dialecte éolien, Dijon 1903, p. 62 ss.; E. Schwyzer, Gr. Gr.2, II, p. 508. 
-ios e -ion. Así, en los papiros ptolemaicos ${ }^{54}$ encontramos formas del tipo

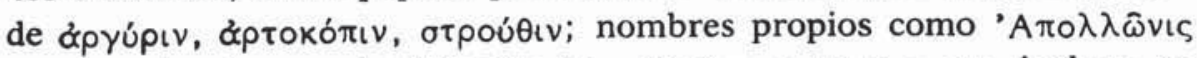
en competencia con el originario, 'A $\pi 0 \lambda \lambda \hat{\omega} v$ เo૬; $\Pi \tau 0 \lambda \varepsilon \mu \alpha i \varsigma$, incluso, en vez de $\Pi \tau 0 \lambda \varepsilon \mu \propto$ đios, etc. También en otros muchos lugares nos ofrece la koiné los mismos resultados, y en laconio reciente tenemos ejemplos de la evolución de -eos, genitivo de singular de los temas en -es a través

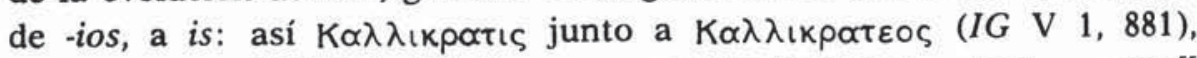
A рібтокротпр (IG V 1, 653 a) -que presenta itacismo: $\eta$ por 1 -, etc. ${ }^{55}$ Por otro lado, Kretschmer ${ }^{56}$ explicó muy bien la composición de nombres propios, que aparecen en inscripciones del laconio reciente, del tipo de $\Sigma(\delta \propto \mu \circ \varsigma$, que poviene de $\Theta \varepsilon \circ \delta \propto \mu \circ \varsigma$, donde se constata claramente la evolución -eo- $>-i o->-i$ - que nos ocupa; y además, expuso acertadamente todos los casos en que tiene lugar la mencionada evolución:

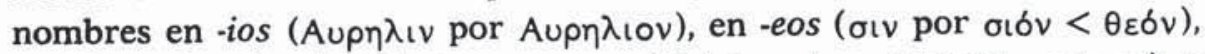

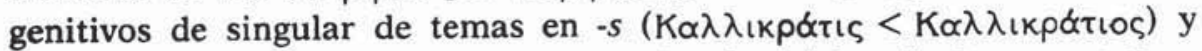
casos en que -eo- se encuentra en interior de palabra $(\Sigma 1 \delta \varepsilon \kappa \tau \alpha \varsigma<\theta \varepsilon \circ-$ $\delta \varepsilon \kappa \tau \alpha \varsigma)^{57}$.

El mismo Kretschmer, sorprendido por la coincidencia de la evolución, constatable en algunos casos, entre el laconio reciente y la koiné, dedujo que el resultado -io- $>-i$ - cuando aparecía en griego helenístico había que interpretarlo como un dorismo en la koiné: «so könnte die vulgärgriechische Zusammenziehung von io zu $i$ ein Dorismus der Koine seins 58 .

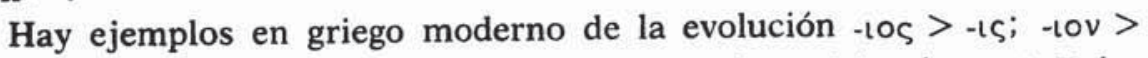

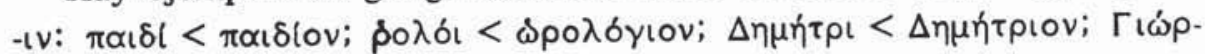
$\gamma \iota \varsigma<\Gamma \varepsilon \omega$ pyıs, etc. ${ }^{59}$.

En conclusión, pues, por lo que a este rasgo respecta, no es peculiar del panfilio, sino que lo reencontramos en la koiné de otras localida-

54 Cf. E. Mayser, Grammatik der griechischen Papyri aus der Ptolomäerzeit, I, 2, pp. 15-16.

55 Cf. P. Kretschmer, «Literaturbericht für das Jahr 1907, Griechisch», Gl 1, 1909, p. 354.

56 Cf. P. Kretschmer, "Literaturbericht für das Jahr 1908, Griechisch», Gl 2 , 1910 , p. 325.

5 Cf. P. Kretschmer, «Literaturbericht für das Jahr 1910, Griechisch», Gl 4, 1913, pp. 320-321.

58 Cf. P. Kretschmer, Literaturbericht für das Jahr 1916, Griechisch», Gl 10 , 1919, p. 227. Cf., asimismo, P. Kretschmer, "Literaturbericht für die Jahre 1922 u. 1923, Griechisch», Gl 14, 1925, p. 203.

59 Cf. A. Thumb, Handbuch der neugriechischen Volkssprache, Estrasburgo 1910 p. 48: «Die Wörter auf - เৎ sind Umbildungen von altgriechischen auf Zahl durch das lateinische Suffix -dpıs (-arius) bedeutend vermehrt worden istn. P. 58: *Die Neutra auf -1 sind eine schon in hellenistischer Zeit vollzogene Umbildung des alten Typen auf - lov". 
des ${ }^{\infty}$. No olvidemos que las inscripciones más antiguas del panfilio son datables en los siglos IV ó III a. J. C. ${ }^{61}$.

Algo similar podríamos decir de las formaciones de femeninos en

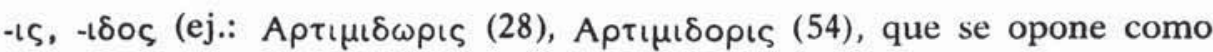

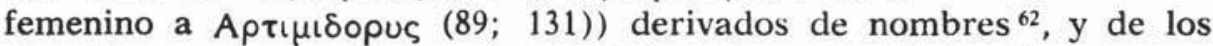
abundantes neutros en $i$-<-ion, del tipo de $\alpha \beta \alpha \tau i(3,6)<{ }^{*} \alpha F \alpha \tau i o v$ o

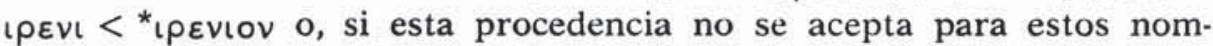

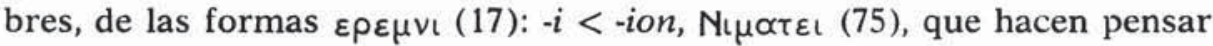
en la misma reducción de -ion en - $i$ que tiene lugar precisamente en los numerosos neutros de la koiné en -ion, diminutivos sin significación de tales. Esta reducción desde la koiné pasa al griego moderno, cuyos dialectos casi en su totalidad -se exceptúa el de las islas del sudeste-

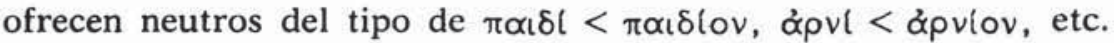

Coincide también el panfilio y la lengua de los papiros ptolemaicos, ejemplo de koiné, en la debilidad de $-v$ al final de palabra.

Otros rasgos comunes al panfilio y a la koiné son: 1) la debilidad

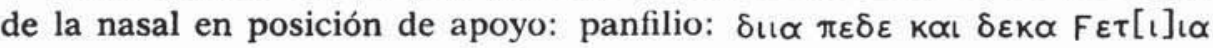

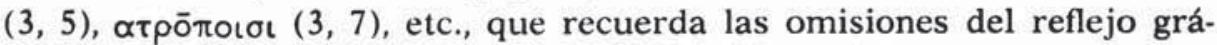
fico de la "nasal de apoyo» en inscripciones, tanto en chipriota - donde la omisión es de regla, p. ej.: pa-ta $(\pi \alpha v \tau \alpha)$ - como en otros dialectos, en los que la omisión es ocasional, p. ej.: át. vú $\phi \bar{\varepsilon}$ (por $v u ́ \mu \phi \eta$ ), etc.; ya desde época imperial nos encontramos con el resultado del griego moderno ( $v u ́ \phi \eta$, \&́$\theta \rho \omega \pi \circ \varsigma$, etc.). 2) La evolución $-\gamma->-y$, que se da ya en panfilio en el siglo IV a. J. C.; p. ej.: $\mu h \varepsilon i \alpha \lambda \alpha(3,5)$, al igual que la forma ática $o \lambda$ เos (del 318 a. J. C.), y que tiene ya en los papiros de la época de los Ptolomeos sus buenos ejemplos (verbigracia: $\sigma \phi \rho \alpha t \delta \omega v$,

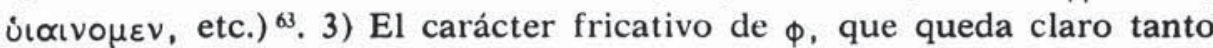
en panfilio $\phi \iota \kappa \alpha \tau \iota)(17 ; 18)<F$ เ $\alpha \tau \iota$ como en las transcripciones latinas de griego $\phi$ por la labiodental $f$, p. ej.: Filippus de $\phi(\lambda \iota \pi \pi \circ \varsigma$. La fricativización de $\beta$, visible en panfilio $h \varepsilon w o \tau \propto \iota \sigma \iota ~(3,7)$, en posición intervocálica, y en koiné, en las transcripciones del latín $u$, p. ej.: $\Lambda \varepsilon i \beta i \alpha$ (lat. Liuia). 4) La desaparición de la oposición de cantidad en el sistema vocálico, que se aprecia en documentos recientes del panfilio - p. ej.:

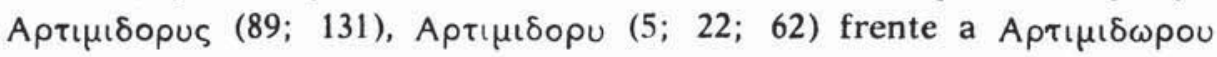

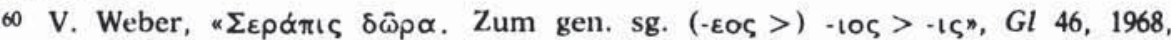
pp. 256-263; cf. p. 262: «...dass wir keinesfalls eine Erscheinung vor uns haben, die der Koine nur innerhalb einer bestimmten, geographisch eng begrenzten Bereiches eigneten.

61 Cf. Cl. Brixhe, o. c., p. 3.

62 E. Mayser, o. c., I, 3, pp. 52-54.

63 E. Mayser, o. c., I, 1, p. 163 ss. 
(73; 79; 88; etc.)-y también, como es bien sabido, en la koiné ${ }^{64} .5$ ) La sonorización de oclusivas sordas procedentes de nasal (en panfilio sólo hay constancia de la evolución $n t>n d$, p. ej.: $\varepsilon \xi \alpha \gamma o \bar{\delta} \iota<\varepsilon \xi \alpha \gamma \omega v \tau \imath$ ( 3 , 16), aunque parece probable que los grupos $m p$ y $n k$ hubieran pasado a $m b$ y $n g$ ). 6) La monoptongación de los diptongos ei y ou en $\bar{e}$ y $\bar{u}$ respectivamente. 7) La similar evolución en panfilio y en la koiné de un sistema acentual preferentemente musical a otro de índole intensiva ${ }^{65}$. 8) El paso de $\theta[t h]$ a fricativa interdental, rasgo que en panfilio es claro a juzgar por el contraste observado entre la grafía $\theta$ en $\Theta \propto v \alpha \delta \omega$ pus (26) y $\tau$ en $\alpha \tau \rho \bar{\pi} \pi$ เ de $d$ en posición intervocálica, que no terminó de imponerse en koiné hasta el paso al griego moderno, pero que, en cambio, parece clara en

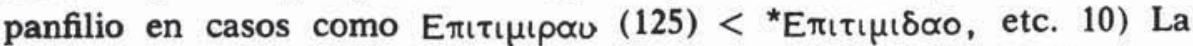

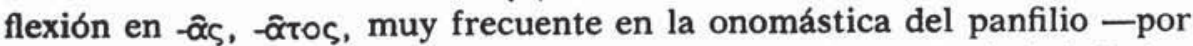
ejemplo: $\Pi \varepsilon \lambda \alpha \varsigma$ (104), $\Pi \varepsilon \lambda \alpha \tau u \varsigma ~(84)-\mathrm{y}$ también en la koiné de las épocas ptolemaica y romana ${ }^{66}$. 11) Los antropónimos masculinos en

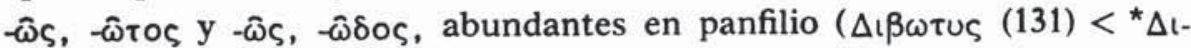

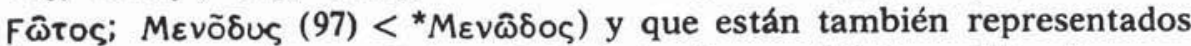
en la onomástica del Egipto helenístico ${ }^{67}$, etc. 12) La utilización con sentido voluntativo del subjuntivo de presente precedido de negación, en vez del subjuntivo de aoristo ( $\mu \bar{\varepsilon} \varepsilon \xi \propto \gamma \bar{o} \delta \mathrm{t} ; 3,16$ y 20 ), etc.

Hay, además, en las inscripciones panfilias rasgos que son claramente debidos a influencia de la koiné en este dialecto y no resultados coincidentes de evoluciones paralelas. Así, por ejemplo, se registran en panfilio casos de reintroducción parcial de formas antiguas por influjo

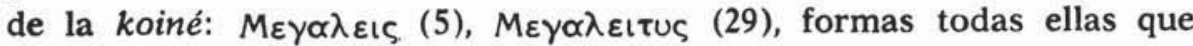
exhiben reintroducción de $-\gamma$ - frente a la forma antigua, epicórica y más

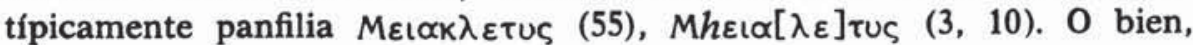

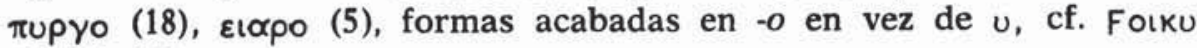
$(3,14 ; 17)$, etc.

Difícil dialecto, pues, el panfilio, que, junto a grandes arcaísmos, como los que ya hemos visto, ofrece tremendas innovaciones que se reencuentran en la koiné, bien como resultado de evoluciones paralelas

64 Cf. E. Mayser, o. c., I, 1, p. 97. F. Blass-A. Debrunner, Grammatik des neutestamentlichen Griechisch, Göttingen $1949_{8}$, p. 15. O. Hoffmann - A. Debrunner A. Scherer, Historia de la lengua griega, trad. esp., Madrid 1973, p. 315. Cl. Brixhe, o. c., p. 27.

65 E. Schwyzer, Gr. Gr.2, I, pp. 392-394. A. Hoffmann-A. Debrunner-A. Scherer, o. c., p. 315 . $\mathrm{Cl}$. Brixhe, o. c., p. 27.

6 E. Mayser, o. c., I, 2, p. 6 ss. Cl. Brixhe, o. c., p. 105

67 Cf. E. Mayser, o. c., I, 2, p. 35. 
independientes en ambas modalidades de lengua, bien debidas a influencia del griego común sobre el dialecto de Panfilia.

Pero, como si todo esto fuese escasa complicación, hay en panfilio indicios de influencia de sustrato de lenguas anatolias, entre los que Cl. Brixhe ${ }^{68}$ cuenta la existencia de un acento de intensidad, la gran frecuencia de casos de metátesis y aféresis, la persistencia de $i \underset{u}{i}$ y tras $i$ y $u$ en hiato, la neutralización de la oposición $o / u$ en final de palabra, la debilidad de la nasal en final de sílaba y el paso de $g$ y $d$, en posición intervocálica, a fricativas.

De todos estos rasgos que Brixhe señala, la mayoría puede, a nuestro juicio, explicarse sin recurrir al sustrato. En cualquier caso, no es esto lo que ahora nos interesa, sino, más bien, tratar de establecer la situación dialectal del panfilio entre los demás dialectos griegos.

Pues bien, de lo que llevamos expuesto pueden entresacarse las siguientes conclusiones: 1) el panfilio es un dialecto en parte muy conservador y en parte muy evolucionado, dos rasgos que pueden explicarse por la situación geográfica del dialecto, apartado del resto de las hablas griegas y en contacto permanente con lenguas extranjeras. 2) Con los dialectos dóricos y eólicos coincide en arcaísmos y elecciones, no en innovaciones, que son las únicas que permitirían establecer la existencia de parentesco específico entre esos dialectos y el panfilio.

La cuestión que ahora se nos plantea de inmediato es la de valorar el carácter probatorio de los rasgos comunes al dialecto que estudiamos y al grupo dialectal «aqueo» (arcadio y chipriota), en el que tradicionalmente se le viene incluyendo.

En el ya aludido trabajo de Metri titulado "Il dialetto panfilio", se señalan isoglosas con los dialectos eólicos laxo sensu (es decir, dialectos eólicos propiamente dichos o lesbio, tesalio y beocio, si se prefiere; dialectos aqueos o eólicos meridionales, como los llama Metri; y finalmente, rasgos eólicos de la poesía homérica). Como para Metri muchos rasgos dialectales son eólicos, se comprende que adopte la conclusión siguiente: el panfilio es un dialecto fundamentalmente eólico *in senso largo ${ }^{\oplus}$ sobre el que se han superpuesto elementos dóricos (particularmente, del cretense). Pero nosotros ya hemos criticado las aparentes conexiones del panfilio con el grupo dialectal eolio. Ahora nos resta, pues, examinar los puntos concretos que el dialecto que estudiamos ofrece en común con los eolismos de la lengua homérica - según la clasificación y denominación de Metri- y con el grupo dialectal «aqueo» (concretamente, con el arcadio y con el chipriota).

$68 \mathrm{Cl}$. Brixhe, o. c., p. 146.

69 P. Metri, o. c., p. 112. 
En común con la lengua homérica señala Metri ${ }^{70}$ dos rasgos del panfilio: 1) el empleo de la preposición $\delta ı \nless$ con acusativo para expresar la extensión en el espacio o en el tiempo, y 2) el aoristo mixto o aoristo sigmático de flexión temática. Del primero contamos con los ejemplos

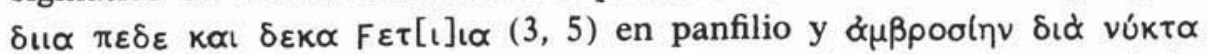
(II. II 57) en Homero. Pensamos que, si bien es cierto que este uso de la preposición $\delta \iota \alpha ́$ sólo se registra en panfilio y en poesía, no tiene nada de sorprendente ni por lo que se refiere al caso acusativo al que acompaña ni por lo que atañe al sentido de $\delta$ ı́́ en el referido sintagma, pues sigue siendo el mismo que conserva el preverbio $\delta เ \alpha$ - en $\delta\llcorner\alpha \beta \alpha l v \omega$, etcétera. Es, pues, un arcaísmo "1. En cuanto al aoristo llamado mixto o, mejor, aoristo sigmático de flexión temática, se localizan en el corpus de inscripciones panfilias tres ejemplos seguros, a saber: $\varepsilon \beta 0 \lambda \alpha \sigma \varepsilon \tau u$

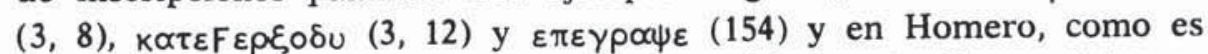

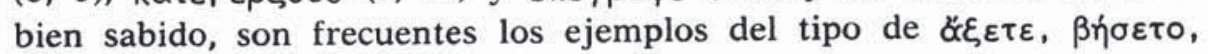
etcétera ${ }^{72}$. Ahora bien, a nuestro juicio, estamos ante un proceso de elección que puede ser antiguo, ya que en indoeuropeo dentro de la

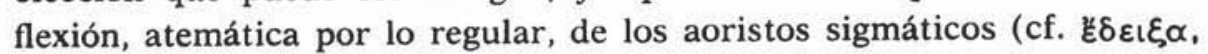
a. esl. zna < znas) algunas personas estaban provistas de vocal temática $^{73}$ (cf. そ̌ $\varepsilon \varepsilon ı \xi \varepsilon, d i ̄ x i t$, a. esl. -sŭ, -somŭ, -sově, etc.).

Llegamos, por fin, a la valoración de las isoglosas que unen al panfilio con el grupo dialectal "aqueo" propiamente dicho, o sea, el arcadio y el chipriota.

Éstas son ${ }^{74}:$ 1) El cierre de $e$ en $i$ ante nasal $m$ o $n$, rasgo en el que el panfilio coincide con el arcadio y con el chipriota. 2) El cierre de $-\varnothing$ en $-u$, rasgo que comparten panfilio, arcadio y chipriota. 3) La aparición de una semiconsonante tras $i$ o $u$ en hiato, rasgo en que coinciden micénico y chipriota, y esporádicamente el arcadio, con el panfilio. 4) La debilidad de la nasal al final de una sílaba, rasgo que puede tal vez poner en conexión al panfilio con el chipriota. 5) La desinencia verbal

70 P. Metri, o. c., p. 109.

71 E. Schwyzer, Gr. Gr.2, II, p. 452: «Beim Akkusativ der Erstreckung geht $\delta$ เ $\alpha$ zunächst der Verwendung beim Genitiv parallel. So rein lokal nur noch bei den Dichtern: «durch-hindurch...».

72 E. Schwyzer, Gr. Gr.2, II, p. 788: «Eine Anzahl von scheinbaren thematischen Aoristformen (bes. Imperativen und Indikativen) gehört zum Futurum (bzw. zu dem diesem zugrunde liegenden Präsens mit $s »)$. P. Chantraine, Morphologie historique du grec, París $1961_{2}$, p. 182: «S'agit-il de formes de vieux désidératifs? Ou d'une forme artificielle de la langue épique?».

73 K. Brugmann, Abrégé de Grammaire comparée des langues indo-européennes, trad. fr., París 1905, p. 571: «Pourtant il y avait à la même date, dans des conditions impossibles à déterminer avec exactitude, des formes à voyelle thématiquex.

74 Cf. P. Metri, o. c., p. 109; Cl. Brixhe, o. c., pp. 145-146. 
-nai que sirve para formar infinitivos atemáticos tanto en arcadio y chipriota como en panfilio. 6) Uso del dativo en función de ablativo, rasgo en que coincide el pantilio con el arcadio, el chipriota y, quizás, también con el micénico.

1) En cuanto al primer punto hemos de señalar que el cierre de $c$ en $i$ ante nasal se produce en arcadio en la preposición y preverbio $\varepsilon \nu(-)$, que, por consiguiente, pasa a $\iota v(-), y$, en interior de palabra, en

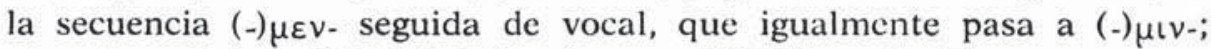
ejemplos: Schw. 665 A $3-4$ (Orcómeno, 360-350 a. J. C.) Epxoutvioıs; Schw. 661, 20 (Mantinea, siglo v a. J. C.) $\propto \pi \varepsilon \chi \circ \mu \nu v \circ \varsigma ; ~ S E G$ XI, 1123

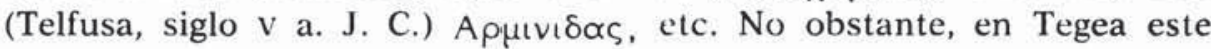
cierre se limita únicamente a la preposición y preverbio $\iota v(-)$ y al participio $\mu$ tvovo๙ı (Schw. 657, 49-50; 51; Tegea, 324 a. J. C.). Las restantes formas que presentan la secuencia $-\mu \varepsilon v$ - mantienen efectivamente la $e$ inalterada.

En chipriota el cierre de $e$, además de aparecer en la preposición y preverbio $\iota v(-)$ y en formas en que se esperaría la secuencia $-\mu \varepsilon v$ -

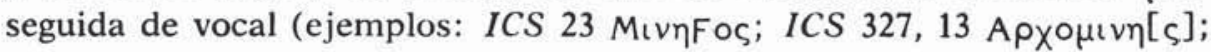

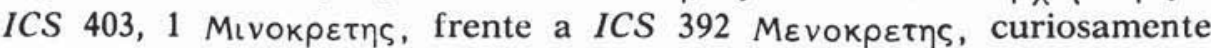
ambas formas son de Abido; por el contrario: ICS 217, 18 A $\mu \varepsilon v i y \alpha$;

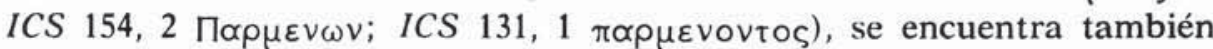
en el pronombre enclítico $\mu \mathrm{t}(<\mu \varepsilon)$-cf., por ejemplo, ICS 234, 2 y 235, 2, en Citio- frente a $\mu \varepsilon v$ (ICS 261, Golgo); también se observa el cierre en $M \iota \gamma \alpha \lambda \alpha \theta \varepsilon \omega$ (ICS 327, 5), etc. Cabe señalar además la vacilación $e / i$ en las formas $E \delta \propto \lambda \operatorname{Iov}(\operatorname{ICS} 217,1$ y 27-8; 220, 1; 218, 2; etc.)

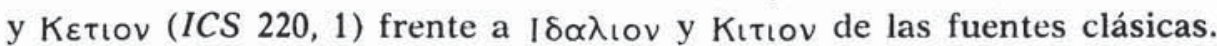

La situación del panfilio, por consiguiente, difiere un tanto de la del arcadio y la del chipriota. Sólo coinciden exactamente en el cierre de $e$

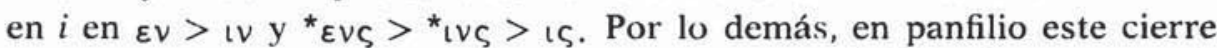
vocálico se produce ante $m$, por ejemplo $\delta \iota{ }_{0}(3,24)$, si es que realmente es evolución de ${ }^{\star} \delta \varepsilon \dot{\varepsilon} \mu \omega v$. Igualmente se localizan en panfilio for-

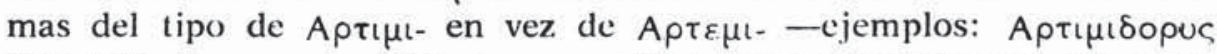

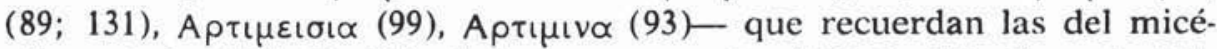
nico atimite (PY Un 219,5) frente a atemito (PY Es 650,5) y timitija (PY Jo 438, 24) frente a temitijo (PY Ac 1278).

Si se tiene en cuenta que la articulación de una nasal lleva consigo un estrechamiento del canal expiratorio, a la altura del paladar blando, motivado por el descenso del velo del paladar, habrá que ser muy cautos a la hora de valorar la coincidencia en el cierre de en en in, que, además de localizarse en arcadio, chipriota y panfilio, se constata 
en cretense central ( $\downarrow$ en Vaxo y Eleuterna; is en Vaxo). No hay que olvidar que hay casos esporádicos de cierre de otra vocal de abertura media $(o)$ ante una nasal ( $m$ o $n$ ) en otros dialectos, por ejemplo: lesbio

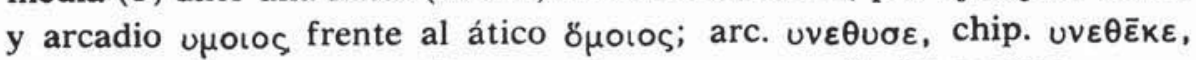
frente a arc.

El cierre de $e$ en $i$ en ${ }^{*} \varepsilon v \varsigma>$ เ del panfilio es, desde luego, anterior a la segunda oleada de alargamientos compensatorios.

A la vista de los hechos la postura más aconsejable es admitir que una serie de dialectos (micénico, arcadio, chipriota, panfilio, cretense) coinciden en mostrar cierta vacilación de timbre vocálico $e / i$ que en micénico es, sin duda, antigua y en los demás dialectos, posiblemente, también.

2) El segundo rasgo, el cierre de $-\varnothing$ final en $-u$, es mucho más claro por estar más generalizado en los dialectos. Por consiguiente, la coincidencia de los tres dialectos en este punto tiene mayor valor probatorio. En arcadio toda ${ }^{*} \varnothing$ final de palabra procedente del griego común 0 resultante de la caída de oclusiva final de palabra pasa a - $u$, a excepción de la de la forma outo (Schw. 665 A 25 y $\mathrm{C}^{1}$ 4, Orcómeno, 360-350 a. J. C.), único ejemplo de conservación de una * $\varnothing$ final antigua (no - 0 de $n$, por ejemplo: $\delta \varepsilon \kappa o$ ) inalterada en arcadio, tal vez debido a su

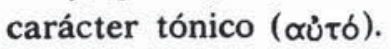

En chipriota toda $-\delta$ final antigua ha pasado a $-u$ excepto en un par de casos en que aflora el arcaísmo: ICS 306, 2 e-ke-ra-to; ICS 357 Kuтрауорао.

Por lo que se refiere al panfilio, contamos con abundante documentación del cierre; así, $\varepsilon \beta 0 \lambda \alpha \sigma \varepsilon \tau u(3,8), u<\delta(3,13)$, genitivos en $-\alpha v$ $(<\bar{a} o)$, etc. Pero hay que añadir que este cierre se ha extendido y ha llegado a afectar a - $o$ acompañada, en sílaba final de palabra, de - $n$ o $-s$ :

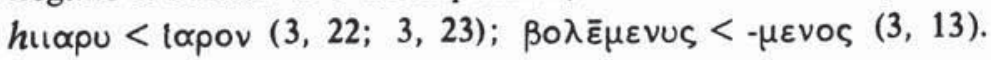

3) Veamos ahora la aparición de un glide $(i, u)$ tras $i, u$ en hiato. Hay ejemplos claros de ambos fenómenos en panfilio; así huхpoı ı (3, 1), $\alpha \phi u \varepsilon \vee \alpha \mathrm{L}(3,6), \Sigma \varepsilon \lambda \cup F u \circ \varsigma(3,3)$, etc.; estos fenómenos se columbran también en micénico y chipriota, donde aparecen notados con mayor o menor regularidad (en arcadio únicamente en las formas EuFaเv[ō],

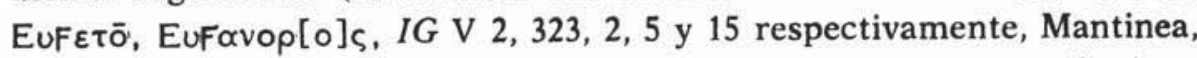
425-385 a. J. C.). Pero lo que es innegable es que estamos ante fenómenos demasiado generales (en argivo, en efecto, leemos По $\lambda \iota \alpha \delta$ (Mnemosyne 57, 1929, p. 208), en jonio Tnuo (IG I 2, 205, 10), en la Calcidica Sufo (Schw. ad 793) y en Eretria (IG XII 9, 1273/74, I 6) y en 
Etolia ( $I G$ IX $1^{2}, 152$ d) $\delta \cup F \varepsilon$ ) como para que los convirtamos en rasgos característicos y definidores de específicos grupos dialectales.

4) En panfilio, desde los primeros textos, no se nota en la escritura la nasal que en interior de palabra se encuentra ante consonante, ni la nasal en posición final absoluta de palabra: ejemplos: $\pi \varepsilon \delta \varepsilon<\pi \varepsilon v \tau \varepsilon(3$, 5); $\pi \circ \lambda_{\iota} \kappa \propto \iota ~\left(\pi \delta \lambda_{\iota} \nu \kappa \propto l\right) ~(3,24-25)$; ambos fenómenos dejan traslucir claramente un hecho: la debilidad articulatoria de la nasal al final de sílaba ${ }^{75}$. En chipriota tampoco se nota una nasal que cierra sílaba, cuando sigue una oclusiva (ejemplo: ICS 217, 3 atoropose öv $\theta \rho \omega \pi \circ \varsigma$ ), pero el mismo fenómeno ocurre aquí y allá en los diferentes dialectos

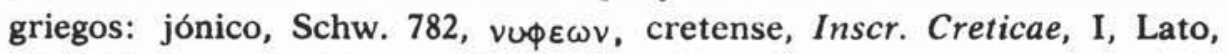
n. $5,32, \pi 0 \pi \pi \alpha \nu(<\pi \circ \mu \pi \bar{\alpha})$, etc.

5) La desinencia atemática - $v \propto \iota$ de infinitivo se atestigua en panfilio -así, $\alpha \phi \iota \varepsilon v \propto \iota(3,6)$ - al igual que en jónico-ático y arcadio-chipriota. Ahora bien: conviene recordar que estamos ante un hecho de elección, del que sólo podemos inferir cierto contacto entre los dialectos que lo comparten. Es, en cambio, mucho más significativa la coincidencia en el vocalismo radical del verbo que significa 'querer' que muestran panfilio $\beta o \lambda \varepsilon \mu \alpha$, arcadio $\beta o \lambda o \mu \alpha$, lesbio $\beta o \lambda \lambda \circ \mu \alpha \iota$ y jónico-ático $\beta o u ́ \lambda o-$ $\mu \alpha$.

6) En cuanto al uso del dativo en función de ablativo, es un hecho en panfilio, en una de cuyas inscripciones leemos $\varepsilon \xi \delta \varepsilon \phi \cup \sigma \varepsilon \lambda \alpha(3,19)$; también lo es en arcadio, dialecto en que se registran ejemplos como

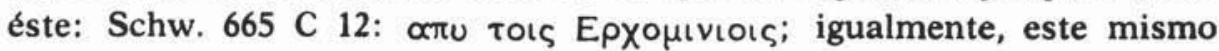
fenómeno se comprueba en chipriota (por ejemplo ICS 217, 5-6 $\varepsilon \xi, \tau \omega \iota$

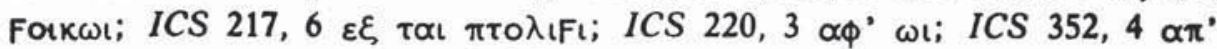

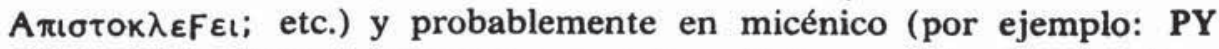
Ea 52 a pa-ro-da-mo). Creemos que no hace falta recurrir, para explicar este hecho, a postular raros sincretismos, sino que estamos ante un fenómeno de elección antiguo.

Por consiguiente, los hechos que acabamos de presentar, si bien unos más que otros, en su conjunto nos mueven, no obstante, a considerar al panfilio dialecto, genéticamente en sus orígenes, afín al arcadio, al chipriota y al micénico. En un segundo término, también al jónico-ático. Es, pues, una derivación del llamado griego oriental que se separó del grupo cuando todavía no habían triunfado definitivamente en éste deter-

75 Cl. Brixhe, o. c., p. 65. 
minadas innovaciones, como la asibilación de $t i$ en $s i$, la simplificación de $s s$ en $s$, la fijación más o menos estricta del vocalismo resultante de las sonantes vocálicas, etc., y, en cambio, compartía otras con determinados dialectos (o protodialectos) del mismo grupo, como el arcadio y el chipriota (por ejemplo: el cierre de -o final en $-u$ ).

Dicho de otro modo: el panfilio es el dialecto resultante de una modalidad dialectal del griego del II milenio a. J. C., que en los arcaísmos que conserva coincide con el dorio, y en algunas innovaciones, antiguas, en cambio, con el micénico, arcadio y chipriota. Como los arcaísmos no prueban en dialectología parentesco específico sino genérico, y sí lo prueban, en cambio, las innovaciones, habrá que deducir: 1) que el panfilio se desgaja de un grupo de dialectos paramicénicos del cual van a surgir con el tiempo el arcadio y el chipriota; 2) que en el segundo milenio a. J. C. el antepasado del panfilio era muy próximo a los del arcadio y chipriota así como al micénico, y era, además, dialecto puente entre todos los que habían asibilado - $t i$ en -si (micénico, protoarcadiochipriota, protojónico-ático, protolesbio) y los en este punto más arcaizantes (protodorio, protoeolio), sin olvidar que debieron existir modalidades dialectales de compromiso entre ambos grupos, como, por ejemplo, el dialecto o dialectos que sirvieron de base al llamado «aqueo épico» ${ }^{76}$, que, como es sabido, conserva $-t i$ junto a $-s i$, $s s$ al lado de $s$,

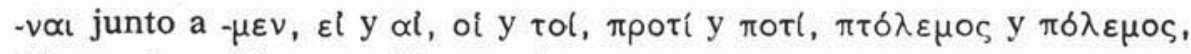

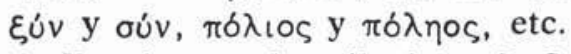

En el segundo milenio a. J. C. hubo probablemente varios tipos de variedades dialectales del griego; unas eran más arcaizantes, conservadoras (de ellas van a surgir los dialectos dorios, el tesalio y el beocio del primer milenio a. J. C.); otras, en cambio, más innovadoras: de ellas tenemos una muestra en el segundo milenio a. J. C.: el micénico; y en el primero, el jónico-ático, el arcadio-chipriota y el lesbio. Entre unas y otras hay que colocar tanto a los dialectos o dialecto de que se nutre el «aqueo épico», como el antecesor del panfilio en el segundo milenio a. J. C.

Esta es nuestra opinión frente a la tradicional más comúnmente aceptada, a saber: que el panfilio es un dialecto aqueo que ha sufrido el efecto de un superestrato dorio. Creemos haber mostrado suficientemente que los rasgos en apariencia dorios del panfilio son arcaísmos, elecciones, o, en último término, fenómenos de carácter general que se dan en panfilio y dorio como meros desarrollos paralelos sin interferen-

\footnotetext{
76 F. R. Adrados, "Micénico, dialectos paramicénicos y aqueo épicon, EMERITA 44, 1976 , pp. $65-113$; cf. p. 72 ss.
} 
cia alguna. En cambio, hemos visto cómo las innovaciones, que sí son decisivas a la hora de establecer grupos dialectales, indican que el panfilio pertenece originariamente al grupo dialectal denominado "griego oriental» (que comprende, además, al micénico y al "aqueo épico», así como al protolesbio, protojonio y protoarcadio-chipriota), y que es el más arcaizante de dicho grupo (en muchos rasgos más arcaizante que el mismo micénico), razón por la que ocupa una posición intermedia entre este grupo llamado "griego oriental" y el conocido como "griego occidental».

A. López Eire y A. Lillo Alcaraz 